1998-10-01

\title{
Isophote-Based Interpolation
}

\author{
Bryan S. Morse \\ morse@byu.edu \\ Duane Schwartzwald
}

Follow this and additional works at: https://scholarsarchive.byu.edu/facpub

Part of the Computer Sciences Commons

\section{Original Publication Citation}

B. S. Morse and D. Schwartzwald, "Isophote-based interpolation," in IEEE International Conference on Image Processing (ICIP), pp. 227-231, October 1998.

\section{BYU ScholarsArchive Citation}

Morse, Bryan S. and Schwartzwald, Duane, "Isophote-Based Interpolation" (1998). Faculty Publications. 639.

https://scholarsarchive.byu.edu/facpub/639 


\title{
Isophote-Based Interpolation
}

\author{
Bryan S. Morse and Duane Schwartzwald \\ Department of Computer Science, Brigham Young University \\ 3361 TMCB, Provo, UT 84602 \\ \{morse,duane\}@cs.byu.edu
}

\begin{abstract}
Standard methods for image interpolation are based on smoothly fitting the image intensity surface. Recent edgedirected interpolation methods add limited geometric information (edge maps) to build more accurate and visually appealing interpolations at key contours in the image. This paper presents a method for geometry-based interpolation that smoothly fits the isophote (intensity level curve) contours at all points in the image rather than just at selected contours. By using level set methods for curve evolution, no explicit extraction or representation of these contours is required (unlike earlier edge-directed methods). The method uses existing interpolation techniques as an initial approximation and then iteratively reconstructs the isophotes using constrained smoothing. Results show that the technique produces results that are more visually realistic than standard function-fitting methods.
\end{abstract}

\section{Introduction}

Standard methods for resolution enhancement (interpolation) are often based on attempts to mathematically fit a function (e.g., piecewise constant, linear, cubic, etc.) to existing pixels. While higher-order interpolants can achieve reasonable results, they still show artifacts of the original discretization, as indeed all interpolation methods must to some degree.

One of the most perceptible artifacts of insufficient image reconstructions from lower-resolution images is noticeable boundaries between the original pixels. This causes what should be smooth contours in the image to be ragged-an effect commonly known as the "jaggies" (see Figure 1a).

This paper presents a method that performs interpolation by reconstructing geometric properties of the original image. Instead of approaching interpolation as "fitting the function", this method approaches it as "fitting the geometry": reconstructing the geometry of the original image's intensity level curves (spatial curves of constant intensity). This reconstruction is done using non-linear diffusion of in- tensities within an initial (jagged) approximation to smooth image contours to match the contours of the original. Thus, it directly attacks one of the the most perceptible artifacts of image reconstruction and causes the interpolated image to preserve smooth contours in the original.

While edge-directed interpolation algorithms (see [1] for an example) have used geometric features (edges) to create smoother subpixel approximations of image edges, they raise two questions: how do you define the edges of interest, and what do you do elsewhere? Rather than trying to extract specific image curves for smooth reconstruction, the method proposed here works by reconstructing smooth approximations of all of the image isophote contours simultaneously.

\section{Interpolation as Isophote Reconstruction}

One of the more visually significant geometric properties of images are their level curves or isophotes (curves of constant intensity). These curves are what give images their perceptual contours. Although isophotes don't capture all geometric information that one might want in analyzing image content [6], reconstruction of the isophotes produces a convincing reconstruction of the image.

The method presented in this paper attempts to reconstruct the image isophotes by explicitly measuring and minimizing their curvature at each point in the reconstructed image, given the constraints of the initial pixel intensities. Notice that this is spatial curvature of the isophote, not curvature (second derivatives) of the image function with respect to intensity. Therein lies the essence of the methodmatching of visually-significant geometric properties, not function-analytical properties of the image.

\section{Reconstruction Artifacts and Isophotes}

Figure 1 shows an example of the effects of interpolation on isophote curvature and length. If a simple blackand-white edge (1a) is interpolated using a bicubic fit (1b), the result still shows the original pixel pattern. The effects of this interpolation on the isophotes can be seen by exam- 
ining individual level curves in the interpolated image (1c). Clearly, the isophotes are not straight, even though the original edge was.

Performing the same operations on a less-sharp edge (intermediate greylevels in the transition) shows similar results. Even though each original isophote (1d) is straight, the isophotes of the resulting interpolation (1e) are not (1f). Notice also that the isophotes seem to exhibit a tendency to (a)

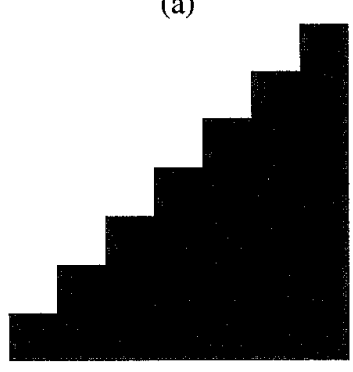

(b)

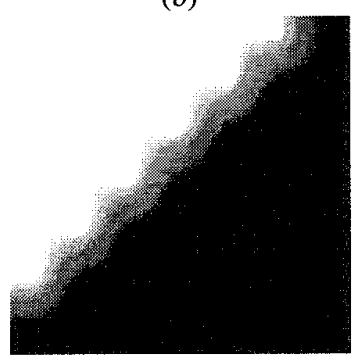

(c)

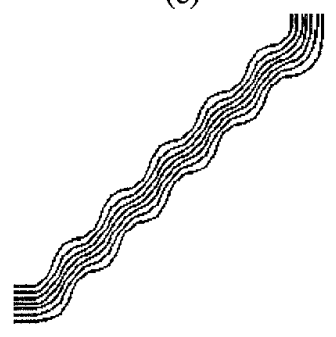

(d)

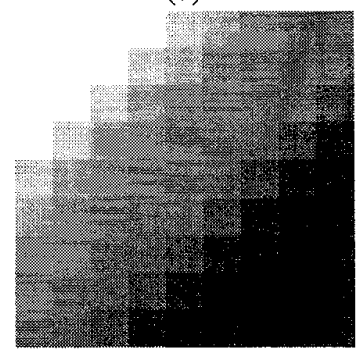

(e)

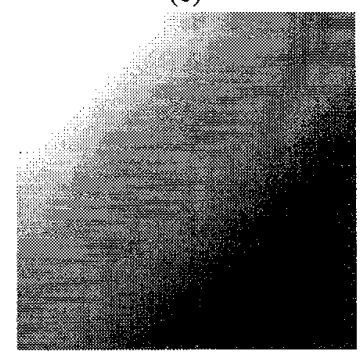

(f)

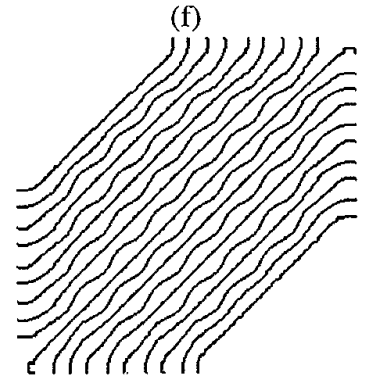

Figure 1: Isophote reconstruction errors introduced by interpolation. When a black-and-white diagonal edge (a, magnified) is interpolated using bicubic interpolation (b), it still shows artifacts of the original sampling grid. Examination of individual level curves (c, eight levels) shows how the level curves are themselves still jagged instead of smooth. When a similar edge with more gradual greylevel transition (d, magnified) is interpolated with bicubic interpolation (e), the isophotes (f, 20-levels) are again jagged. be attracted to the horizontal and vertical alignment of the original pixel grid-an expected artifact of interpolation.

\section{Isophote Reconstruction Algorithm}

The preceding examples suggest another approach to image interpolation: smooth fitting of each isophote curve based on the original image constraints. This can be phrased as a reconstruction/optimization problem: find the set of isophotes that

- Preserve isophote topology and ordering,

- Preserve intensities at "known" (original pixel) positions, and

- Are each as smooth as possible.

An illustration of this is shown in Figure 2.

Although no closed-form solution is known for these constraints, this optimization can be approached iteratively in a gradient-descent fashion:

1. Begin with an approximation of the "optimal" interpolation by using currently available interpolation techniques, and

2. Iteratively minimize isophote curvature while maintaining "anchors" at the original pixel positions.

\subsection{Initial Interpolation}

As with all gradient-descent approaches to optimization, the initial approximation is important. Here, we may use any of the existing interpolation methods as our first approximation. We have tested our method with initial approximations derived through bilinear interpolation, bicubic interpolation, and even simple pixel replication. In each case, the initial interpolation is improved by constrained isophote smoothing.

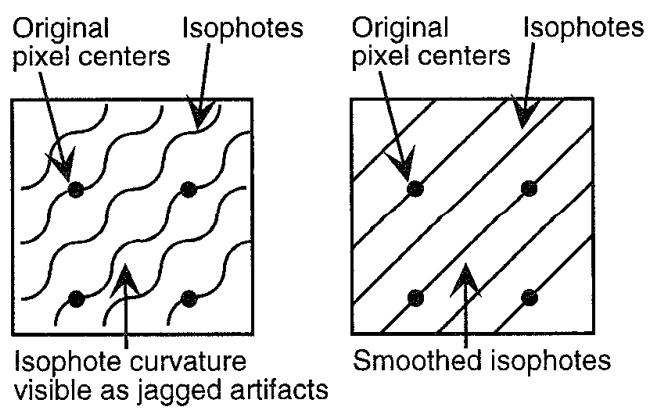

Figure 2: Illustration of constrained isophote smoothing 


\subsection{Constrained Isophote Smoothing}

At first glance, manipulation of the isophotes requires explicitly finding and fitting each isophote, much as individual edges must be found and fitted in edge-directed approaches [1]. However, such explicit curve-fitting is not necessary.

Recent research by Alvarez, Lions, and Morel [2], Sethian, Malladi, and Kimmel $[3,4,5,6]$ and others have demonstrated techniques for performing isophote curve smoothing by considering the surface of all level curves (the intensity surface of the image) and at each pixel solving for the change in pixel intensity that causes the corresponding isophote to "evolve" in a desired fashion [7]. They have shown that this type of "flow" can be used to perform edgepreserving smoothing, noise removal and other image enhancement, and shape evolution and description. Here, we use a constrained form of this curve evolution to reduce the artifacts of interpolation. Whereas other applications of level-set smoothing have attempted to enhance the original image, we are attempting to reconstruct the original image, only with higher sampling.

Specifically, we use a form of this isophote curve evolution that causes the isophotes to move with a speed proportional to the curvature at each point (curvature flow):

$$
I_{t}=-\kappa\|\nabla I\|
$$

where $\kappa$ denotes the isophote curvature calculated by

$$
\kappa=\operatorname{div}(\nabla I /\|\nabla I\|)=-\frac{I_{x}^{2} I_{x x}+2 I_{x} I_{y} I_{x y}+I_{y}^{2} I_{y y}}{I_{x x}^{2}+I_{y y}^{2}}
$$

This provides two of our three goals: preservation of isophote topology and isophote length/curvature minimization, but we must add an additional constraint to preserve intensities at the original pixel locations:

$$
I_{t}=\left\{\begin{array}{cl}
0 & \text { if on an original pixel location } \\
-\kappa\|\nabla I\| & \text { otherwise }
\end{array}\right.
$$

This is similar to constrained curve evolution as presented in [3].

\section{Results}

An example of this isophote-reconstruction technique is shown in Figures 3 and 4.

Figure 3 shows the same image magnified three times using replication (to show the original resolution), bilinear, bicubic, and isophote-based interpolation. Each of the traditional interpolation methods show pixelization artifacts. Each also shows typical expected results: bilinear interpolation shows fewer pixelization artifacts but appears blurred; bicubic interpolation produces a sharper result but with increased jaggedness.

These jagged artifacts are significantly smoothed in the isophote-based interpolated image. The result starts with and keeps the sharpness of bicubic interpolation while smoothing the isophotes to reduce jagged pixelization artifacts. These effects are most visible at places with sharp edges: the eye, the top of the earring, the cheeks, etc. These can be better seen in magnified portions of the corresponding interpolated images (Figure 4).

\section{Discussion}

The primary advantage of this approach is smooth reconstruction of the individual isophote contours without explicit calculation or representation of each such contour. By directly attacking perceivable artifacts rather than focusing on fitting the data, this method produces more visually appealing interpolations (magnifications) of images than traditional methods. This has potential applications in web-based image distribution, high-resolution printing, and compression.

\section{References}

[1] Jan Allebach and Ping Wah Wong. Edge-directed interpolation. In IEEE International Conference on Image Processing, pages 707-710, 1996.

[2] L. Alvarez, P. L. Lions, and J. M. Morel. Image selective amoothing and edge detection by nonlinear diffusion. SIAM J. Numer. Anal., 29(3):845-866, 1992.

[3] James A. Sethian. Level Set Methods. Cambridge University Press, 1996.

[4] Ravikanth Malladi and James A. Sethian. Image processing:flows under min/max curvature and mean curvature. Graphical Models and Image Processing, 58(2):127-141, 1996.

[5] Ravikanth Malladi and James A. Sethian. A unified approach to noise removal, image enhancement, and shape recovery. IEEE Transactions on Image Processing, 5:1554-1568, 1996.

[6] Ron Kimmel, Ravi Malladi, and Nir Sochen. Images as embedded maps and minimal surfaces: Movies, color, and volumetric medical images. In Computer Vision and Pattern Recognition, 1997.

[7] S. Osher and J. A. Sethian. Fronts propogating with curvature dependent speed: Algorithms based on Hamilton-Jacobi formulation. J. Comput. Phys., 79:12-49, 1988. 


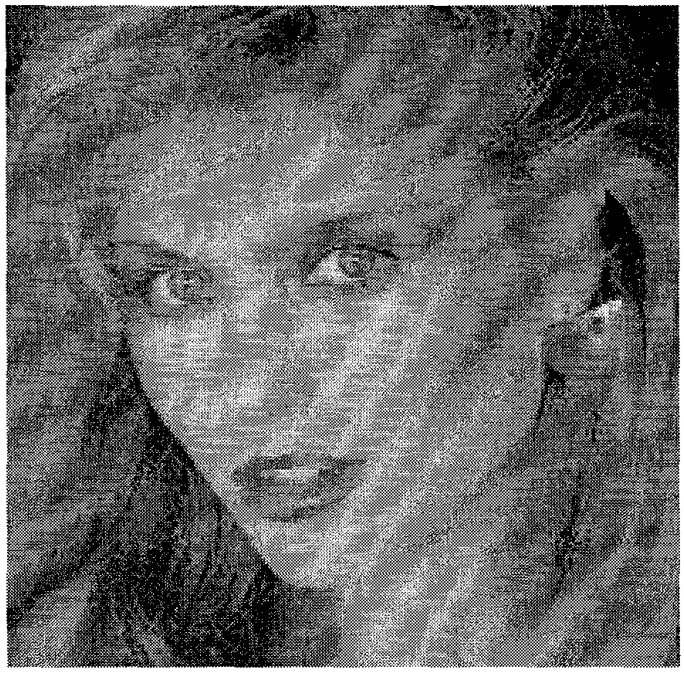

replication

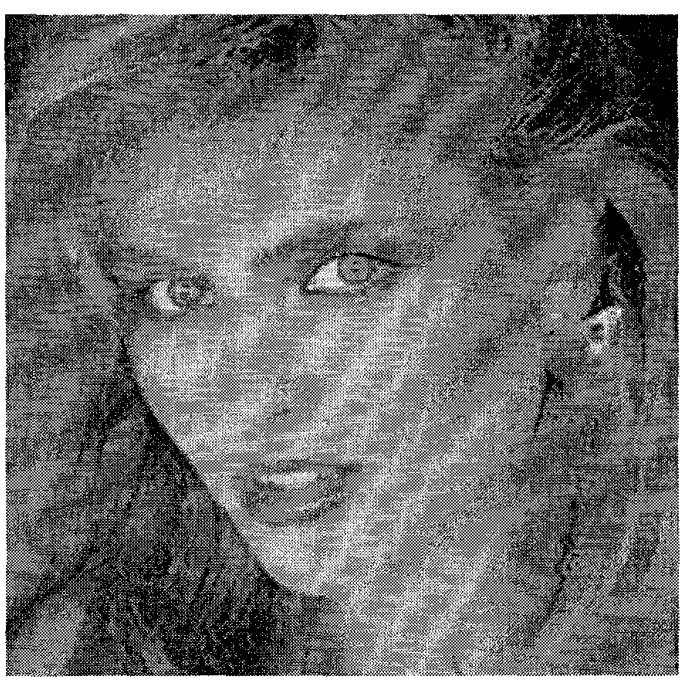

bicubic

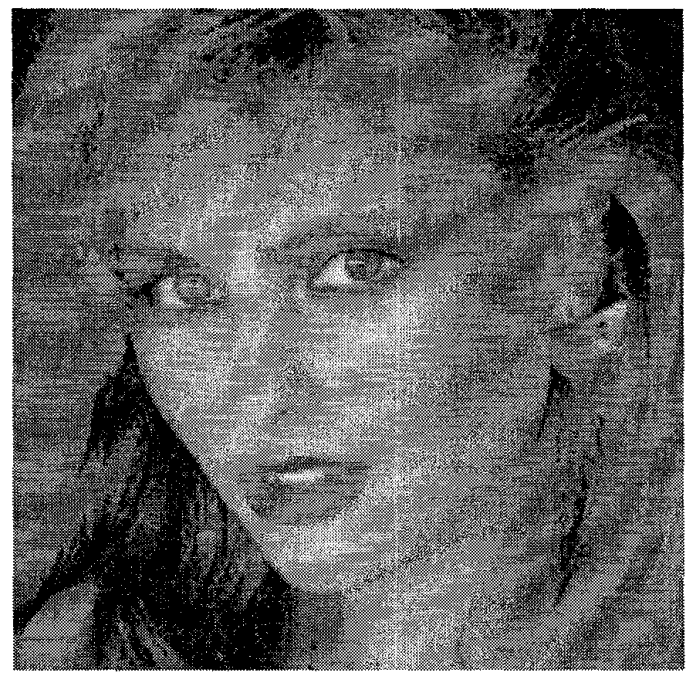

bilinear

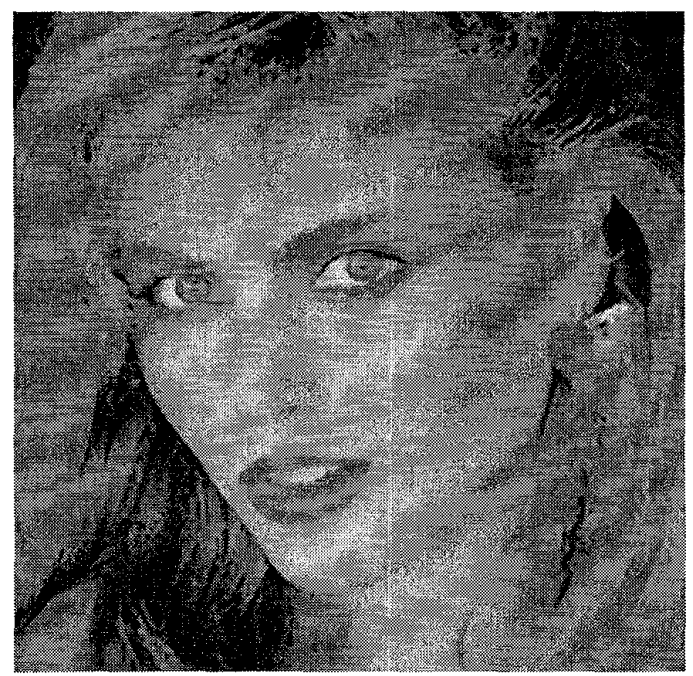

isophote-based

Figure 3: Results of isophote reconstruction. The original image magnified 3x using pixel replication (upper left), bilinear interpolation (upper right), bicubic interpolation (lower left) and isophote reconstruction (lower right). 


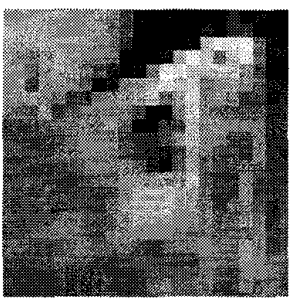

replication

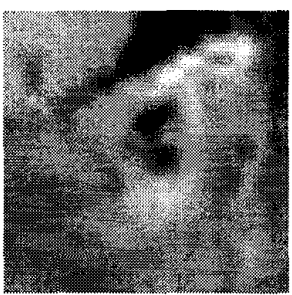

bicubic

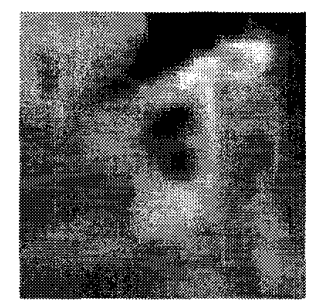

bilinear

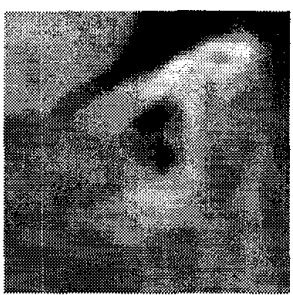

isophote-based

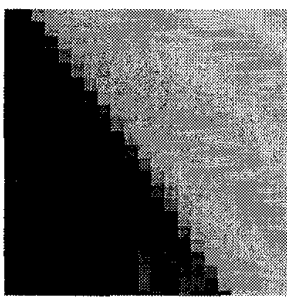

replication

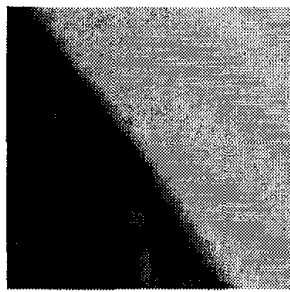

bicubic

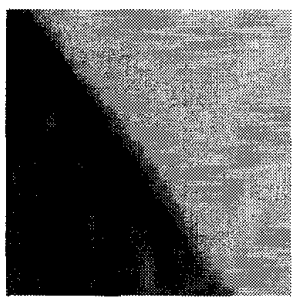

bilinear

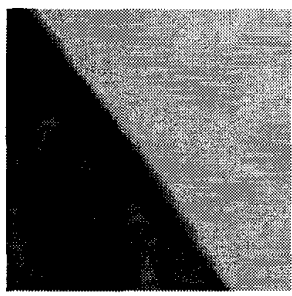

isophote-based

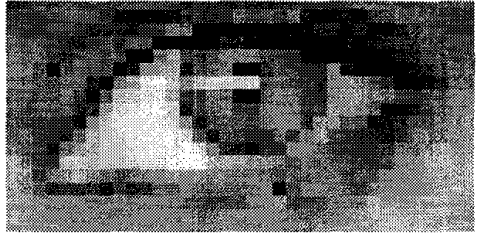

replication

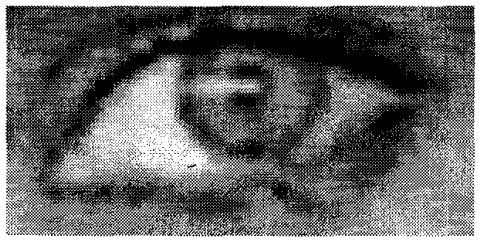

bicubic

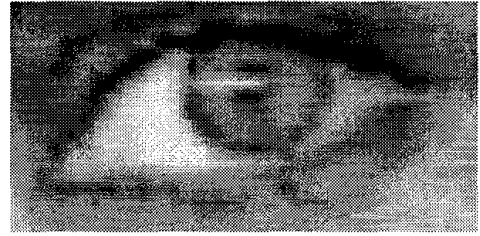

bilinear

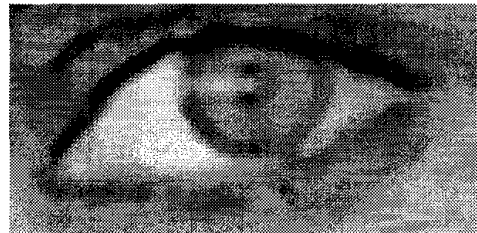

isophote-based

Figure 4: Results of isophote reconstruction. TOP LEFT: upper edge of the earring in the corresponding (replicated, bilinear, bicubic, isophote reconstruction) images from Figure 3. TOP RIGHT: left edge of the cheek in the corresponding images. Bоттом: right eye in the corresponding images. 